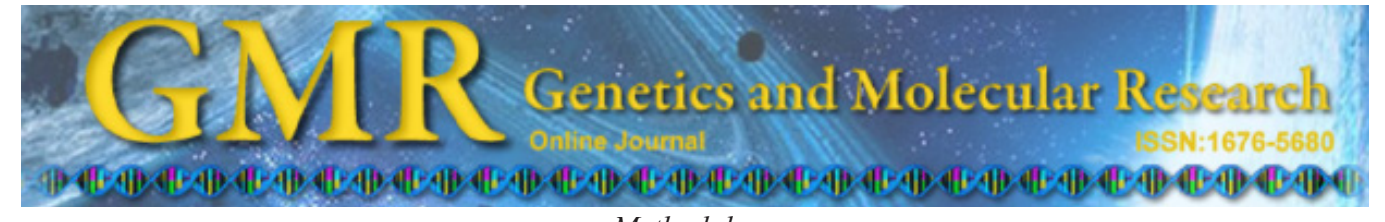

\title{
Methodology \\ Identification of 18 genes encoding necrosis-inducing proteins from the plant pathogen Phytophthora capsici (Pythiaceae: Oomycetes)
}

\author{
B.Z. Feng, P.Q. Li, L. Fu, B.B. Sun and X.G. Zhang \\ Department of Plant Pathology, Shandong Agricultural University, \\ Shandong, China \\ Corresponding author: X.G. Zhang \\ E-mail: zhxg@sdau.edu.cn / sdau613@163.com
}

Genet. Mol. Res. 10 (2): 910-922 (2011)

Received January 7, 2011

Accepted February 16, 2011

Published May 17, 2011

DOI 10.4238/vol10-2gmr1248

\begin{abstract}
Phytophthora capsici is an aggressive plant pathogen that affects solanaceous and cucurbitaceous hosts. Necrosis-inducing Phytophthora proteins (NPPs) are a group of secreted toxins found particularly in oomycetes. Several NPPs from Phytophthora species trigger plant cell death and activate host defense gene expression. We isolated 18 P. capsici NPP genes, of which 12 were active during hypha growth from a Phytophthora stain isolated from pepper (Capsicum annuum) plants in China. The 18 predicted proteins had a sequence homology of $46.26 \%$. The 18 Pсnpp sequences had a conserved GHRHDWE motif and fell into two groups. Eleven sequences in group 1 had two conserved cysteine residues, whereas the other seven sequences in group 2 lacked these two cysteine residues. A phylogenetic tree was constructed on the basis of the alignment of the predicted protein sequences of 52 selected NPP genes from oomycetes, fungi and bacteria from Genbank. The tree did not rigorously follow the taxonomic classification of the species; all the NPPs from oomycetes formed their own clusters, while fungal sequences were grouped into
\end{abstract}


two separate clades, indicating that based on NPPs, we can separate oomycetes from fungi and bacteria, and that expansion of the NPP family was a feature of Phytophthora evolution.

Key words: Oomycete; Necrosis-inducing Phytophthora protein; Phytophthora capsici; Horizontal gene transfer

\section{INTRODUCTION}

Like fungi, oomycetes have a global distribution and prosper in diverse environments. The oomycetes belong to the kingdom Stramenopile and contain a lot of pathogenic species (Baldauf et al., 2000). The genus Phytophthora contains a large number of phytopathogens, such as $P$. capsici, P. infestans, P. sojea, and so on. And most Phytophthora species are the cause of blight, crown rot, as well as stem, leaf, and fruit lesions on many plants. Moreover, the Phytophthora pathogen has been identified as a devastating pathogen on solanaceous and cucurbitaceous hosts including pepper, cucumber, eggplant, squash, pumpkin, tomato, melon, and zucchini (Lamour and Hausbeck, 2004). Sporangia and/or oospores develop in the lesions, resulting in a powdered-sugar appearance on the surfaces of fruits. Infected fruits quickly break down both in the field and postharvest.

Many pathogens have been reported to secrete elicitor proteins, which are recognized by the defense system in the plant. Elicitors are signaling molecules that play an important role in determining host specificity and disease development for compatible and incompatible interactions (Pare et al., 2005). In fact, elicitors are connected to a series of proteins that cause cell death at the infection site, thereby limiting or slowing down disease (Garcia et al., 2007). Although the necrosis can prevent biotrophic growth of pathogens, the induction of such condition can be beneficial to saprotrophic pathogens (Qutob et al., 2002).

NPPs, namely necrosis-inducing Phytophthora proteins, are also called NLPs (Nep1like proteins), because the protein shares substantial sequence similarity with the first discovered member (named Nep1), which was defined as the necrosis and ethylene-inducing protein isolated from Fusarium oxysporm f.sp. erythroloxyli in liquid cultures (Bailey, 1995). NPPs represent a new class of necrotic elicitors.

Genes encoding NPPs, or the proteins themselves, have been detected in superfamilies in eukaryotic and prokaryotic organisms. The NPPs are not found in plants, animals, but they often occur in fungi, bacteria and also in oomycetes. Oomycetes, particularly many Phytophthora species such as $P$. infestans, $P$. parasitica, $P$. aphanidermatum, $P$. sojea, and $P$. ramorum form NPPs (Pemberton and Salmond, 2004). Although NPPs are present in phylogenetically distant organisms, the NPP sequence has remained a remarkably conserved domain called the necrosis-inducing Phytophthora protein 1 (NPP1) (Fellbrich et al., 2002). There is a hepta-peptide (GHRHDWE) and some conserved cysteine residues are present in every sequence. According to the cysteine residue number and position, NPPs are classified into two groups (Gijzen and Nurnberger, 2006). Dean et al. (2005) found that Magnaporthe grisea has the NPP genes of these two groups. To date, monocots are apparently not affected by NPPs, but it has been reported that many NPPs are able to trigger numerous dicotyledonous plant defense responses, necroses and cell death (Fellbrich et al., 2002; Qutob et al., 2002; Bailey et al., 2005). Some organisms, like Verticillium dahliae can secrete NPPs (VdNEP) that induce cotton wilting (Wang et al., 2004). In tobacco leaves, Nep1 caused an increase of ethylene production, indicating that necrosis could be an indirect effect of 
this hormone (Jennings et al., 2000). However, in some plants necrosis induction was not accompanied by ethylene emission, suggesting that other mechanisms may be involved. Studies with $P$. parasitica showed that Nep1 induced superoxide anions production and salicylic acid-dependent resistance gene PR1 expression (Fellbrich et al., 2002). Additionally PsojNIP was linked to the transition from biotrophic to the saprotrophic phase in P. sojea (Qutob et al., 2002). Remarkably, it was observed that the crystal structure of an NLP from Pythium aphanidermatum exhibited structural similarities to cytolytic toxins produced by marine organisms, suggesting that this protein contributed to host infection by plasma membrane destruction and cytolysis (Ottmann et al., 2009). However, the reason why Phytophthora species have a large NPP gene family and the roles these genes play during infection process are still unclear.

The phylogenetic relationship between NPP sequences from different organisms has been analyzed by several authors. Notably, all these authors suggested that horizontal gene transfer occurred during NPP gene evolution, because bacterial NPP genes within a phylogram were not monophyletic, but interspersed with fungal sequences (Pemberton and Salmond, 2004; Gijzen and Nurnberger, 2006; Garcia et al., 2007). On the other hand, an alternative explanation was raised. Garcia et al. (2007) indicated that NPPs were ancient genes, and underwent duplication and evolution, resulting in the division of two types of genes (types I and II) before the division in eukaryotes and prokaryotes. In this case, some organisms that did not use NPP protein such as plants and animals may have lost these genes.

In this study, we showed the presence of NPPs in a high-virulent $P$. capsici strain SD33 isolated from China. A total of 18 NPP genes were cloned and characterized in this strain, 12 of which were apparently active in SD33 mycelia. The sequence alignment of these 18 NPPs delineated two groups. Eleven members in group 1 had two conserved cysteine residues, whereas the other seven sequences in group 2 were lacking these two cysteine residues. All the 18 predicted function proteins displayed high sequence homology with a conserved GHRHDWE motif. The phylogenetic tree was constructed on the basis of the alignment of predicted protein sequences of 52 selected NPP genes from oomycetes, fungi and bacteria. All the NPPs of oomycetes were clustered together, while fungal sequences were grouped into two clades, indicating that NPPs were able to separate oomycetes from fungi and bacteria, and that fungi and bacteria would have acquired these genes by horizontal gene transfer.

\section{MATERIAL AND METHODS}

\section{Phytophthora capsici strain}

A highly virulent $P$. capsici strain, SD33, was used as described previously (Feng et al., 2010) and maintained at $25^{\circ} \mathrm{C}$ on $10 \%$ V8 juice agar medium as described in Tyler et al. (1995).

\section{Isolation of Pcnpp genes and sequence analysis}

The GHRHDWE motif was searched for in the released $P$. capsici genome sequence (http://genome.jgi-psf.org/PhycaF7/PhycaF7.download.html). The candidate amino sequences were determined through manual revision and checked by submitting sequences to the online SMART software (http://smart.embl-heidelberg.de/) for conserved function domain analysis. The orthologs of the reported NPP gene in other oomycte genomes, including $P$. sojea, $P$. 
infestans, and P. parasitica, were downloaded from NCBI. Also NPP genes from reported species in fungi and bacteria were downloaded from NCBI. Most of the available complete NPP amino acid sequences were multialigned using Clustal X 2.0.

To amplify NPP orthologous in the SD33 strain, a series of primers were designed using the Primer Express 3.0 software according to the identified sequences in the JGI database (as shown in Table 1). Genomic DNA of SD33 was extracted from hyphae grown in 10\% V8 liquid medium according to the protocol described by Tyler et al. (1995). Polymerase chain reaction (PCR) parameters were as follows: $94^{\circ} \mathrm{C}$ for $4 \mathrm{~min}, 35$ cycles of $94^{\circ} \mathrm{C}$ for $1 \mathrm{~min}, 55^{\circ} \mathrm{C}$ for $30 \mathrm{~s}$, $72^{\circ} \mathrm{C}$ for $1 \mathrm{~min}$, and a final extension at $72^{\circ} \mathrm{C}$ for $10 \mathrm{~min}$, and minor adjustments were made to amplify different genes. The PCR products were cloned in T3-vector and confirmed by sequencing.

Nucleotide and amino acid sequence homology searches were compared with the sequences in the NCBI-BLAST program (http://www.ncbi.nlm.nih.gov/).

Table 1. Primers used for Pcnpp gene cloning.

\begin{tabular}{|c|c|c|}
\hline Primer name & Primer sequence & Application \\
\hline $7756 \mathrm{PF}$ & ATGCAACTACGTGCCTTCATCTCT & Pcnpp 1 \\
\hline $7756 \mathrm{PR}$ & TTAAGTGTAGTACGCGTTAGCTAGTTTA & \\
\hline $23292 \mathrm{PF}$ & ATGAAATTCGTCGTTTTCCTCTGTG & Pcnpp 2 \\
\hline 23292PR & CTAGAAGGGCCAGGCCTTGTCCAG & \\
\hline $71103 \mathrm{PF}$ & ATGAACCTTCTGGGATTCCTCGCC & Pcnpp3 \\
\hline 71103PR & TTAGAATGGCCAAGCCTTACCCAA & \\
\hline $43883 \mathrm{PF}$ & ATGTACTCTTGGTACTTCCCCAAG & Pcnpp 4 \\
\hline 43883PR & ATTCAGATTCCACTGTGGAAAGAAGC & \\
\hline $70852 \mathrm{PF}$ & ATGAAGTTCAAACCCCAACTGCAC & Pcnpp 5 \\
\hline $70852 \mathrm{PR}$ & CTAGAAGGGCCAGGCCTTGTCCAG & \\
\hline $24573 \mathrm{PF}$ & ATGAGGTTTACCACCATCTTCTGG & Pcnpp6 \\
\hline $24573 \mathrm{PR}$ & TTAAAACGGCCAGGCGTTTTCAAT & \\
\hline $68295 \mathrm{PF}$ & ATGTACATGTGCACCTTTGCCATC & Pcnpp 7 \\
\hline 68295PR & TTAGAACGGCCAAGCCTTGTCCAGT & \\
\hline $73591 \mathrm{PF}$ & ATGAGGCTCAGTATCGCCTTGGGCG & Pcnpp 8 \\
\hline 73591PR & TCATTGAAAGGGCCAAGCTTTGGC & \\
\hline $78535 \mathrm{PF}$ & ATGAGGCTCTTCGCTTTCCTATGG & Pcnpp9 \\
\hline $78535 \mathrm{PR}$ & TTAGAACGGCCAAGCCTTGTCCAGT & \\
\hline $23459 \mathrm{PF}$ & ATGTTCAAGACGTTCATTATCGCTG & Pcnpp 10 \\
\hline 23459PR & CTACTGGTACCAGGCGTTCGCGAGC & \\
\hline 20844PF & ATGGAGCCCCTGAATATAAG & Pcnpp 11 \\
\hline 20844PF & TTAGAAGGGCCAAGCCTTC & \\
\hline $21024 \mathrm{PF}$ & ATGACCGACAGTAAAAACACCGTTACAGCT & Pcnpp 12 \\
\hline $21024 P R$ & CTATTTTTTTTCGCCAAATGGCCAGGCTTTAC & \\
\hline $69004 \mathrm{PF}$ & ATGTACTCGTGGTACTTCCCTAAAGATTCG & Pcnpp 13 \\
\hline 69004PR & CTATTTTTTTTCGCCAAATGG & \\
\hline $70605 \mathrm{PF}$ & ATGTACTCTTGGTATTTCCCCAAGGATTCT & Pcnpp 14 \\
\hline 70605PR & TTAATCAAACGGCCAGGCCTTGTTGAGTTT & \\
\hline 78817PF & ATGTACTCGTGGTACTTCCCAAAGGACTCA & Pcnpp 15 \\
\hline 78817PR & TTAGAAGGGCCAAGCCTTCTCCAGCTTCGG & \\
\hline $86540 \mathrm{PF}$ & ATGTACTCGTGGTATTTCCCCAAGGATTCC & Pcnpp 16 \\
\hline 86540PR & CTAAAAGGGCCAAGCCTTGTCCAACTTGG & \\
\hline $68053 \mathrm{PF}$ & ATGTACTCGTGGTACTTCCC & Pcnpp 17 \\
\hline 68053PR & TTAGAAGGGCCAAGCCTTCTCCAGCTTCG & \\
\hline $72101 \mathrm{PF}$ & ATGTACTCTTGGTACTTCCCGAAAGACTCA & Pcnpp 18 \\
\hline 72101PR & TTAAAACGGCCAGGCGTTTTCAATTTTCG & \\
\hline
\end{tabular}

\section{RNA extract and RT-PCR analysis}

SD33 mycelium, grown in 10\% V8 liquid medium for three days, was collected and ground in liquid nitrogen. Total RNA was extracted using a fungal RNA kit (OMEGA) fol- 
lowing manufacturer recommendations. The RNA was quantified by measuring absorbance at $260 / 280 \mathrm{~nm}$ with a spectrophotometer and the quality was examined by electrophoresis on a $1.2 \%$ formaldehyde agarose gel. Ten micrograms total RNA was treated with 4 U Rnase-free DNaseI (TaKaRa) at $37^{\circ} \mathrm{C}$ for $30 \mathrm{~min}$, and then was used for reverse transcription using the Omniscript RT kit (Qiagen). Synthesized cDNA was used for PCR, and the specific primers for PCR were designed using the Primer Express 3.0 software (Table 2). The PCR parameters were as follows: $94^{\circ} \mathrm{C}$ for $4 \mathrm{~min}$, then 30 cycles of $94^{\circ} \mathrm{C}$ for $1 \mathrm{~min}, 53^{\circ} \mathrm{C}$ for $30 \mathrm{~s}$, and $72^{\circ} \mathrm{C}$ for $30 \mathrm{~s}$, and then $72^{\circ} \mathrm{C}$ for $10 \mathrm{~min}$ for a final extension. P. capsici actinA gene was used as control. The PCR products were electrophoresed on $1 \%$ agarose gels and visualized by ethidium bromide staining. Results were obtained from three repeated experiments.

Table 2. Primers used for RT-PCR.

\begin{tabular}{|c|c|c|c|}
\hline Primer name & Primer sequence & Application & Expected size \\
\hline ARTF & GTTGGACTCACGGA AAATCC & Pcnpp 1 & 147 \\
\hline ARTR & GTGGTCAAGGATAACCCAACT & & \\
\hline BRTF & ATGAAATTCGTCGTTTTCCTC & Pcnpp2 & 179 \\
\hline BRTR & AGTTGGGGTTTGAACTTCATC & & \\
\hline FRTF & TCCAAGCTGGAACTATCGAT & Pcnpp3 & 166 \\
\hline FRTR & AGTCCACCACTGGTCTCTCC & & \\
\hline GRTF & ACGACGGCTACAAGAAGT & Pcnpp 4 & 117 \\
\hline GRTR & AGTATCCAGATCGTGGTTGA & & \\
\hline HRTF & GGGCTTGAAAACTACCGG & Pcnpp 5 & 114 \\
\hline HRTR & CAAGAGTACATGATGGCCCAG & & \\
\hline JRTF & ACCACCATCTTCTGGATCAG & Pcnpp6 & 165 \\
\hline JRTR & AGAGTCTTGAGTCTGCGGTT & & \\
\hline KRTF & GACCCAAGATGACAGCAGTA & Pcnpp 7 & 196 \\
\hline KRTR & TAGGAGCTGGAGTAGGTGAC & & \\
\hline LRTF & GTTCGCAGGTACAACAAATT & Pcnpp 8 & 202 \\
\hline LRTR & AGATAGTGACAGGTTCAGGTTG & & \\
\hline MRTF & GACCCAAGATGACAGCAGTA & Pcnpp 9 & 180 \\
\hline MRTR & TGACAGGGTAGGCAAATATG & & \\
\hline ORTF & CTGAGCGATCATGTACTCCT & Pcnpp 10 & 171 \\
\hline ORTR & AGGATAGTAGACCGTGTAGCC & & \\
\hline QRTF & ACCACCAACTCCAGAACCAA & Pcnpp 11 & 177 \\
\hline QRTR & TTTCAACTTCACACCAGCCTT & & \\
\hline RRTF & GGTCGTGATGGAGATGAAAT & Pcnpp 12 & 118 \\
\hline RRTR & GATTATCTTT TCCGTCCAT & & \\
\hline TRTF & GTCGGCTCTTAGTGGTTATT & Pcnpp 13 & 156 \\
\hline TRTR & AGTCCACATGATGAGGTCTTG & & \\
\hline URTF & GGAAGATCTTGGCTGTCA & Pcnpp 14 & 165 \\
\hline URTR & GGTCCTGGTAGTCCCCTC & & \\
\hline XRTF & GCATGCTGGCTACAAGAA & Pcnpp 15 & 137 \\
\hline XRTR & AGGTCCTGAAAAGTCCCA & & \\
\hline YRTF & AGCGTCTACTCCAAGCAA & Pcnpp 16 & 140 \\
\hline YRTR & CATGATGAGGTCCTGGAAGTC & & \\
\hline SRTF & AAAGGACTCACCGTCTACTG & Pcnpp 17 & 150 \\
\hline SRTR & GGCGGGCACGGATTGTACTT & & \\
\hline VRTF & CCGTCATGACTGGGAGCACG & Pcnpp 18 & 162 \\
\hline VRTR & GTCCAGGTCGTGGTTGTAAG & & \\
\hline ActinRTF & GTACTGCAACATCGTGCTGTCC & Actin gene & 250 \\
\hline ActinRTR & TTAGAAGCACTTGCGGTGCACG & & \\
\hline
\end{tabular}

\section{Phylogenetic analysis}

To generate the phylogenetic tree, 34 reported NPP genes (including 23 type I genes and 11 type II genes) (Pemberton and Salmond, 2004; Gijzen and Nurnberger, 2006) and 18 
Pcnpp genes were used (Table 3). Multiple alignment was performed on these 52 sequences by use of Clustal X (2.0). Phylogenetic trees were generated by neighbor-joining, as implemented in PAUP* 4.0 Beta (Sinauer Associates, Sunderland, MA, USA) with the default parameters. Nodal support of the trees was estimated by bootstrapping, with 1000 pseudoreplicate data sets.

Table 3. NPP sequences used in the phylogenetic analysis.

\begin{tabular}{|c|c|c|}
\hline Organism & Accession number & Gene type \\
\hline Aspergillus nidulans & XP_660815 & I \\
\hline A. nidulans & XP_660939 & II \\
\hline A. fumigatus & XM_-743186 & I \\
\hline A. fumigatus & XM_743446 & II \\
\hline Bacillus halodurans & BAB̄04114 & I \\
\hline B. licheniformis & YP_091187 & I \\
\hline Fusarium oxysporum & AAC97382 & I \\
\hline Gibberella zeae & XP_387963 & II \\
\hline G. zeae & XP_391669 & II \\
\hline G. zeae & XP_383570 & II \\
\hline G. zeae & XP_386193 & I \\
\hline Magnaporthe grisea & $\mathrm{XP}_{-}^{-} 362893$ & I \\
\hline M. grisea & XM_366313 & II \\
\hline M. grisea & XM_365630 & II \\
\hline M. grisea & XM_368843 & II \\
\hline M. oryzae & XM_362983 & I \\
\hline Moniliophthora perniciosa & EF114673 & I \\
\hline M. perniciosa & EF109894 & I \\
\hline Neurospora crassa & XM_954671 & II \\
\hline Phytophthora infestans & AY961417 & I \\
\hline P. parasitica & AF352031 & I \\
\hline P. megakarya & AY741083 & I \\
\hline P. megakarya & AY741082 & I \\
\hline P. megakarya & AY741086 & I \\
\hline P. megakarya & AY741088 & I \\
\hline P. sojea & AF320326 & I \\
\hline P. sojea & AAM48171 & I \\
\hline P. sojea & AAM48172 & I \\
\hline Pythium aphanid & AF179598 & I \\
\hline P. aff. vanterpoolii & AAQ89595 & I \\
\hline P. middletonii & AY389162 & I \\
\hline Verticillium dahliae & AAS45247 & I \\
\hline Vibrio pommerensis & CAC40975 & II \\
\hline Streptomyces coelicolor & AL939131 & II \\
\hline
\end{tabular}

NPPs were divided into two types (I and II) according to numbers of cysteine residues in their sequences as described in previous studies (Pemberton and Salmond, 2004; Gijzen and Nurnberger, 2006).

\section{RESULTS}

\section{Isolation of Pcnpp genes and sequence analysis}

Genome-wide identification of NPPs was performed by searching for the GHRHDWE motif in the released $P$. capsici genome sequence. We identified 53 candidate genes containing the conserved GHRHDWE motif. Among these NPPs, 14 were single copies, while the rest were multicopies ranging from 2-12 (see Table S1). A series of primers were designed using the released P. capsici genome sequence (JGI). The PCR product of expected length was ligated with T3-vector in DH5 $\alpha$ and then sequenced in company. A database search confirmed that it was homologous to NPP genes from oomycete, fungi and bacteria. 
A total of 18 Pcnpp gene sequences designated Pcnpp 1 to Pcnpp 18 were identified and submitted to Genbank (accession Nos. HM543167 to HM543184). They were all complete genes, without an intron. As shown in Figure 1, most of them were expressed in hypha, whereas six genes (Pcnpp4, Pcnpp11, Pcnpp 12, Pcnpp 16, Pcnpp 17, and Pcnpp18) appeared to be inactive in hypha grown by RT-PCR analysis.

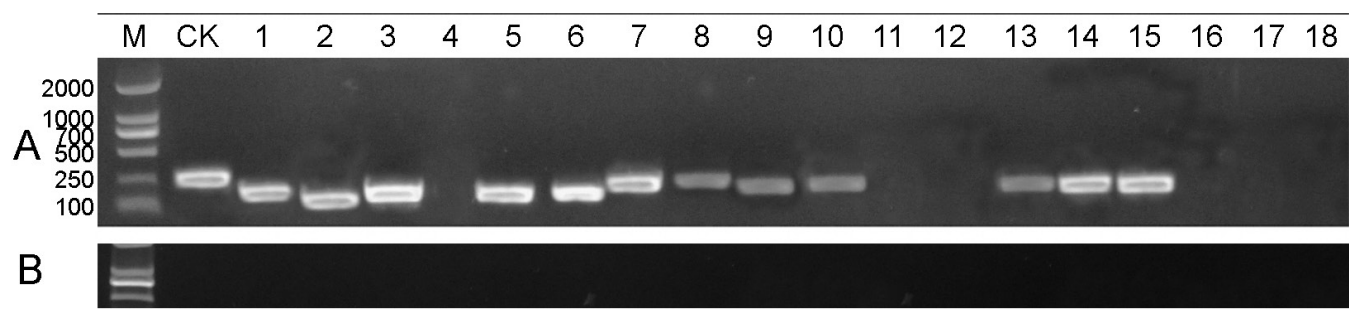

Figure 1. A. RT-PCR analysis of the 18 Pcnpp genes expressed in hyphae RNA. M = DNA marker DL-2000; CK= actinA used as control; lanes 1-18= RT-PCR products: Pcnpp 1 to Pcnpp 18. B. RNA used as template for RT-PCR analysis to detect DNA contamination. Lanes 1-19= there were no bands present, indicating no DNA.

Amino acid sequences were deduced from the open reading frames. The predicted proteins varied in length from 132- to 338-amino acid residues, as a result of $\mathrm{N}$-terminal extension variation in length (Figure 2). Eight genes (Pcnpp 1, Pcnpp2, Pcnpp 3, Pcnpp6, Pcnpp 7, Pcnpp $8, P$ cnpp 9 , and $P$ cnpp 10$)$ contained a signal peptide predicted using SignaLP 3.0 Server as shown in Table 4. Putative monobasic propeptide cleavage sites were present between 17and 20 -amino acid residues in the eight sequences as shown in Table 4.

Table 4. Identities of 18 Phytophthora capsici sequences and signal peptide predictions of the translated proteins.

\begin{tabular}{|c|c|c|c|}
\hline Gene & GenBank No. & Extracellular protein ${ }^{a}$ & SignalP-HMM probability ${ }^{\mathrm{b}}$ \\
\hline Pcnpp 1 & HM543167 & Yes & 0.999 \\
\hline Pcnpp2 & HM543168 & Yes & 1.000 \\
\hline Pcnpp3 & HM543169 & Yes & 1.000 \\
\hline Pcnpp 4 & HM543170 & No & 0.000 \\
\hline Pcnpp5 & HM543171 & No & 0.000 \\
\hline Pcnpp6 & HM543172 & Yes & 1.000 \\
\hline Pcnpp 7 & HM543173 & Yes & 1.000 \\
\hline Pcnpp 8 & HM543174 & Yes & 0.999 \\
\hline Pcnpp 9 & HM543175 & Yes & 1.000 \\
\hline Pcnpp 10 & HM543176 & Yes & 0.999 \\
\hline Pcnpp 11 & HM543177 & No & 0.038 \\
\hline Pcnpp 12 & HM543178 & No & 0.000 \\
\hline Pcnpp 13 & HM543179 & No & 0.000 \\
\hline Pcnpp 14 & HM543180 & No & 0.000 \\
\hline Pcnpp 15 & HM543181 & No & 0.000 \\
\hline Pcnpp 16 & HM543182 & No & 0.000 \\
\hline Pcnpp 17 & HM543184 & No & 0.000 \\
\hline Pcnpp 18 & HM543185 & No & 0.000 \\
\hline
\end{tabular}

${ }^{a}$ A protein sequence is assigned a 'Yes' for extracellular protein if the protein is predicted to be a signal peptide by the SignalP Hidden Markov Model (SignalP-HMM) with a probability greater than 0.900 and the SignalP Network predicted cleavage site between 10 - and 40 -amino acid residues. Otherwise, it is assigned a 'No'. 'Calculated by the SignalP Hidden Markov Model algorithm. 


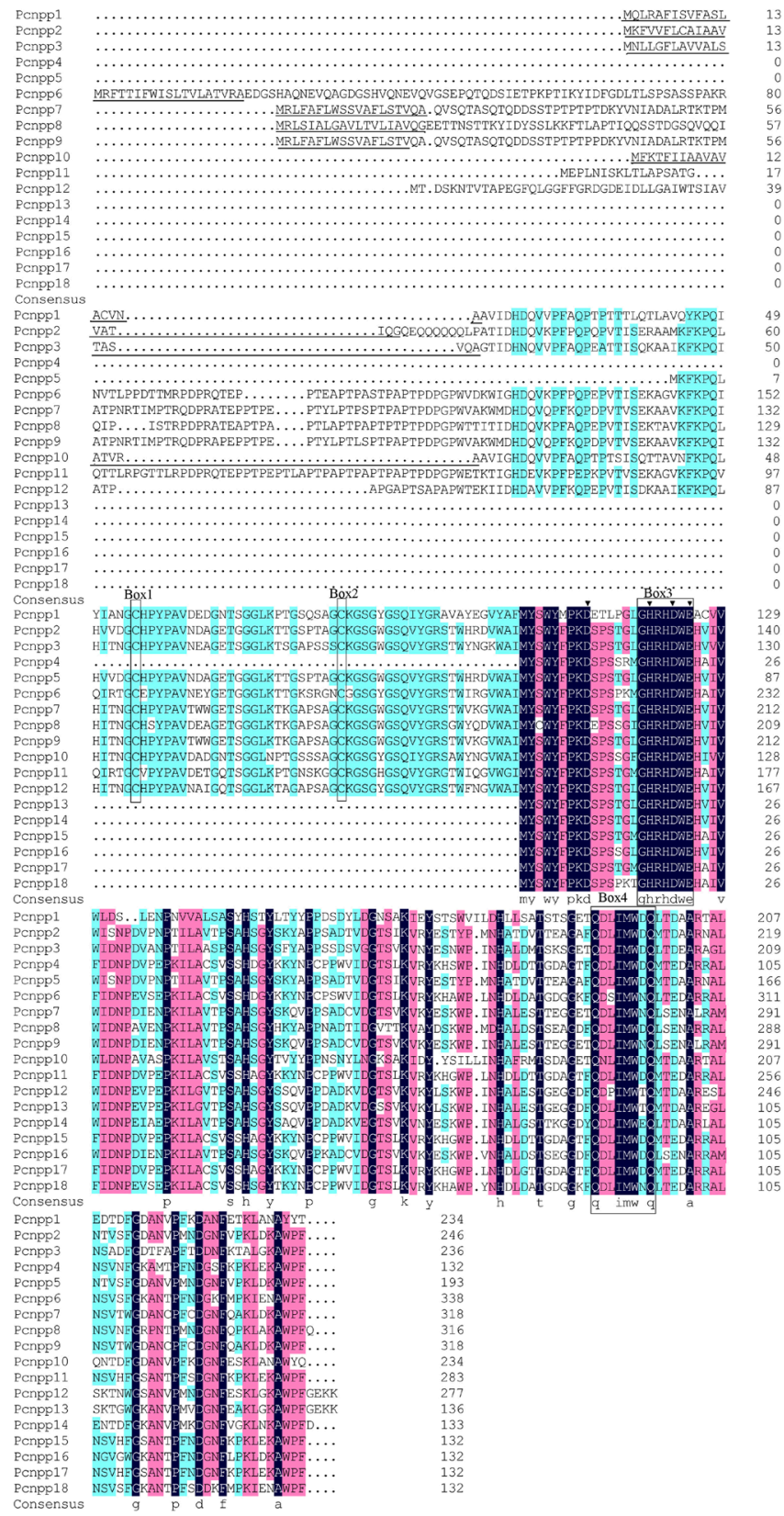

Figure 2. Sequence alignment of the 12 potential Pcnpp genes. Signal peptides of each sequence are in grey, the two conserved cysteines are in Box1 and Box2, the hepta-peptide motif 'GHRHDWE' is in Box3, the C-terminal relatively conserved motif 'QDLIMWDQ' is in Box4. Arrowheads indicate potentially active sites. Signal peptides for each Pcnpp gene are underlined. Dark highlights indicate that the residues are conserved in all NPPs compared, whereas other color highlights denote sequences only conserved in some NPPs. The consensus line shows only those residues that are identical in $100 \%$ of the sequences. 
Sequences and RT-PCR transcripts for these 18 genes, confirmed that 12 of these genes were expressed during in vitro growth. All the 18 predicted proteins displayed sequence homology of $46.26 \%$. The 18 genes fell into two groups. Group 1 had 11 sequences (Pcnpp 1, Pcnpp 2, Pcnpp 3, Pcnpp5, Pcnpp6, Pcnpp 7, Pсnpp8, Pcnpp9, Pcnpp 10, Pcnpp 11, and $P c n p p 12)$ and had two highly conserved cysteine residues, which were numbered $\mathrm{C}^{55}$ and $\mathrm{C}^{80}$ in Pcnpp 1 at the N-terminal extension (Figure 2). By contrast, sequences in group 2 (Pcnpp 4, Pcnpp 13, Pcnpp 14, Pcnpp 15, Pcnpp 16, Pcnpp 17, and Pcnpp 18) were lacking the conserved cysteine residues, and the average length was ca. $400 \mathrm{bp}$. However, all these 18 genes had a conserved GHRHDWE motif and a relatively conserved bexakis-residue QDLIMW at the C-terminal extension. All Pcnpp have four potential enzyme activity sites according to the crystal model by Ottmann et al. (2009). Numbered according to their positions in Pcnpp 1, these residues were $\mathrm{D}^{112}, \mathrm{H}^{120}, \mathrm{D}^{123}$, and $\mathrm{E}^{125}$ (Figure 2). These four residues have been shown to be involved in necrosis lesion formation in NLPs from P. aphanidermatum (Ottmann et al., 2009). Additionally, the Pcnpp had a GC content of $56.6 \%$, which was similar to other Phytophthora NPPs, such as $56.9 \%$ for P. infestans, $59.3 \%$ for P. sojae and $56.6 \%$ for $P$. parasitica (Bae et al., 2005). The identities between Pcnpp sequences and fungi and bacteria were relatively low $(15-44.73 \%)$.

\section{Phylogenetic analysis}

To generate the phylogenetic tree, 34 reported NPP genes (including 23 type I genes and 11 type II genes) and 18 Pcnpp genes were used.

The phylogenetic tree (as shown in Figure 3) showed the relationships among the selected 52 NPPs. The tree was constructed on the basis of alignment of the predicted protein sequences of the selected NPP genes. All oomycete genes were clustered together, while fungal sequences were grouped into two separate clades (Fungi 1 and 2). Bacterial sequences were selected as the outgroup. Unexpectedly, two bacterial sequences (AL939131 and CAC40975) were interspersed with fungal sequences in clade Fungi 2 . In our phylogenetic tree, all the genes in clade Fungi 1 belonged to type I as previously described (Pemberton and Salmond, 2004; Gijzen and Nurnberger, 2006); however, in clade Fungi 2 most genes belonged to type II, except four genes (EF114673, EF109894, AAC97382, and AAS45247). This result was somewhat different from the previous conclusion, which showed that NPPs could be grouped strictly according to the gene type. Remarkably, all the oomycete NPP genes were attributed to type I as described above, and Pcnpp were grouped together with other NPP protein sequences from oomycetes, which was consistent with previous results (Pemberton and Salmond, 2004; Gijzen and Nurnberger, 2006; Garcia et al., 2007).

\section{DISCUSSION}

In this study, we confirmed that there was a large NPP gene family comprising at least 18 NPP genes in P. capsici strain SD33. Although many NPP genes occurred in several Phytophthora species, little was known about these genes at the molecular and biological level in $P$. capsici. Other plant pathogens contained multiple NPP genes, but the size and structure of this gene family was diverse. For example, Magnaporthe grisea and Gibberella zeae possess four NPPs (Dean et al., 2005; Gijzen and Nurnberger, 2006), whereas Aspergillus nidulans and 


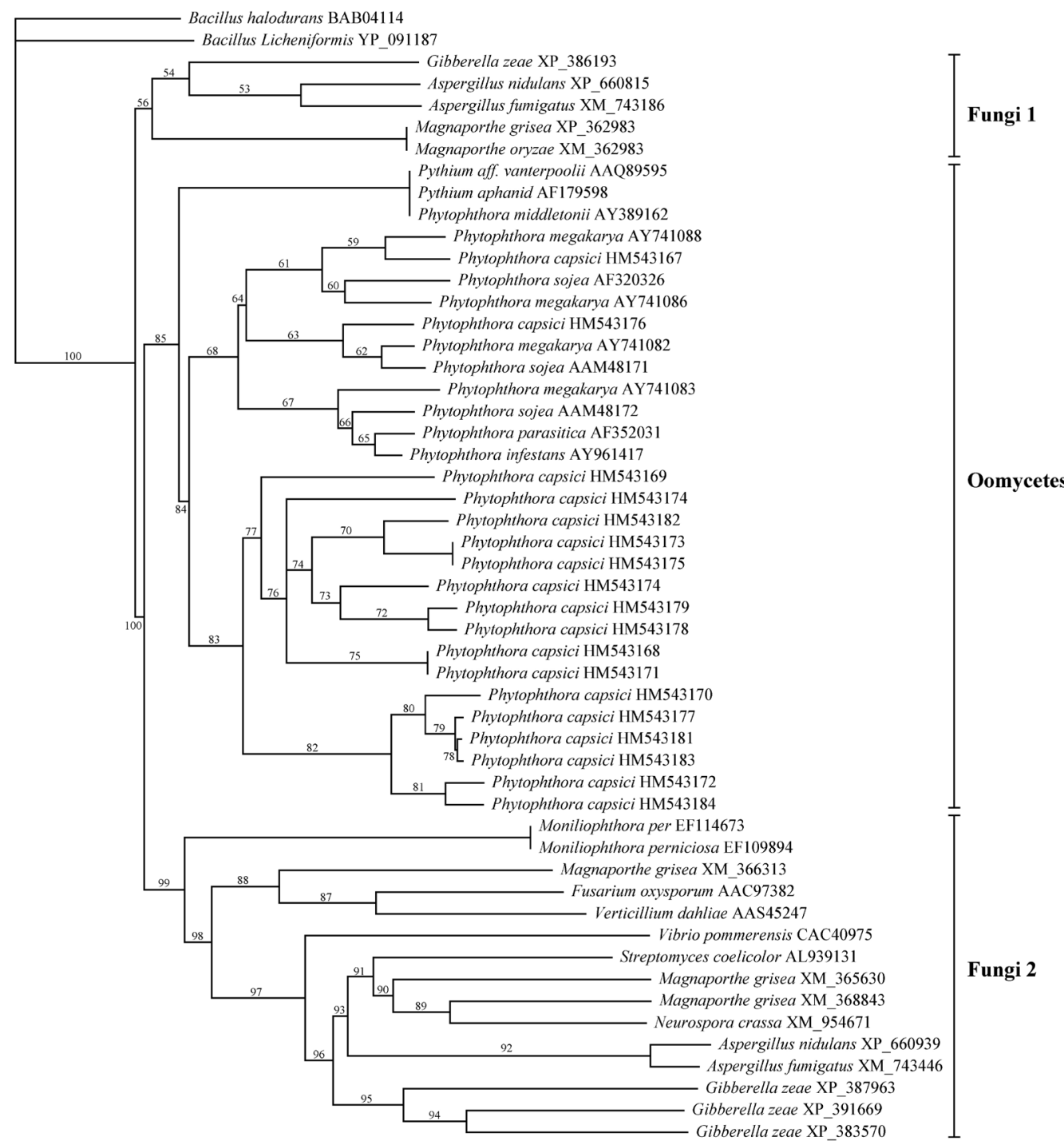

Figure 3. Phylogenetic analysis of the sequences of NPP orthologs from oomycetes and other organisms. The phylogram was generated by the neighbor-joining method as implemented in PAUP* 4.0 Beta. Numbers beside each node indicate bootstrap values as a percentage of 1000 replicates, with the scale bar representing $10 \%$ weighted sequences divergence. The accession number of each gene is shown to the right of the organism.

A. fumigatus each contain two NPP genes (Galagan et al., 2005), and a single gene is present in the Neurospora crassa genome (Galagan et al., 2003). Many bacterial species contain only 1-4 NPP genes in their genome (Pemberton and Salmond, 2004). Genome sequences demonstrate that around 50-60 NPP genes are present in P. sojae and P. ramorum, respectively; but it is believed that more than half of the predicted genes are likely pseudogenes (Tyler et al., 2006). 
In P. megakarya, NPP orthologs have been found organized in clusters and at least six of them seem to be expressed (Bae et al., 2005).

Although a number of possibilities have been proposed about the roles of NPPs, the biological significance of multiple NPPs in plant pathogens is not fully understood. It is likely that NPPs play an important role in the pathogenic progress of Phytophthora (Qutob et al., 2006). Some NPPs are related to pathogenicity during pathogen infection of plants, but NPPs in many fungi and bacteria species are not known to be pathogenic in plants. This suggests that NPPs may have other physiological functions within the pathogen other than their role in plant infection. In contrast, NPP sequences are not found in plant or animal, which suggests that NPPs are involved in the physiological or pathogenic processes of microorganisms.

All Pcnpp grouped together by phylogenetic comparison with NPPs from oomycetes, fungi and bacteria. The results showed that the fungal NPPs containing two or four conserved cysteines did not form separate clades, which were different from previous studies that classified all the fungus NPP genes as type I and II. Fungal NPP sequences were divided into two different groups (Fungi 1 and 2), the type I in Fungi 1 and both types in Fungi 2 (Figure 3 ). This result was different from a former study indicating that all NPPs could be clearly separated according to the number and position of conserved cysteins (Gijzen and Nurnberger, 2006; Garcia et al., 2007). However, the tree was able to better group oomycete NPPs, as shown in Figure 3. The members of the oomycete clade belonged to Phytophthora and Phythium species. They formed three main branches in the oomycete clade, in which all Pcnpp formed a compact cluster, except Pcnpp 1 (HM543167) and Pcnpp 10 (HM543176). This result suggested that the NPP family was a character of oomycete organisms, and was suitable for oomycete classification. On the contrary, it seemed that the NPP genes were not suitable for fungi or bacteria classification. Also there was no monophyletic group containing fungal NPPs or bacterial NPPs, respectively (Figure 3). The phylogenetical distribution of fungi and bacteria might be explained by the horizontal gene transfer mechanism. In fact, the nucleotide composition of certain bacteria was unequal to the genomic average, which suggested that these bacteria got NPP genes from other organisms (Pemberton and Salmond, 2004). In addition, NPPs existed in certain basidiomycetes like MpNEPs in Moniliophthora perniciosa, but could not be detected in other basidiomycete genomes, indicating discontinuity in the evolution of these genes. Moreover, although the codon usage of MpNEPs was similar to that of other M. perniciosa genes, the GC content was much higher than that of other genes, suggesting that these NPP genes were recently introduced in the species by horizontal gene transfer from other organisms (Garcia et al., 2007). On the contrary, Phytophthora NPPs had a GC content and codon usage that were the same as those of NPP genes in other organisms, indicating that these NPPs were ancient gene families in the Phytophthora species. Taken together, these data indicated that they were able to separate oomycete from fungi and bacteria, and that the occurrence of NPPs in fungi and bacteria might be involved in horizontal gene transfer.

Although NPPs showed relatively high homology, these sequences had intraspecific and interspecific diversity in structure and biological performance. In fact, 18 Pcnpp showed structural diversity in this study, and a case in point was that two NPP genes (MpNEP1 and MpNEP2) in M. perniciosa had high similarity but showed different characteristics when applied to tobacco and cacao leaves (Garcia et al., 2007). Maybe this was connected to birth-anddeath evolution that had spent enough time for the divergence of gene duplicates and/or their orthologs in different organisms (Nei and Rooney, 2005). In addition, the percentage identities 
between the deduced amino sequence of Pcnpp and other NPPs ranged from 15 to $84.19 \%$. The highest matches were found with NPPs of Phytophthora, $84.19 \%$ between Pcnpp 1 (HM543167) and P. megakarya (AY741088), and 83.9\% between Pcnpp 10 (HM543176) and P. sojae (AAM48171). And many NPP genes were apparently pseudogenes and occurred in more than one copy in some species. Additionally, these phenomena agreed with the birth-anddeath theory that gene duplication and mutation emerged in an evolutionary process to adapt to different niches. As a result of duplication and mutation process, genes would diverge either in structure or in expression pattern, or be rendered non-functional as pseudogenes, and would be eventually removed from the genome (Garzón-Ospina et al., 2010).

In summary, we reported that an NPP multigene family existed in P. capsici, and that these paralog proteins had diverse physical properties and would be differentially expressed. We also did primary phylogenetic analysis of NPPs from different organisms, indicating that NPP expansion was a feature of Phytophthora. It is possible that the Pcnpp genes play an important role in pepper blight. We are currently studying the necrosis mechanisms, and developing protocols for silencing these genes in P. capsici.

\section{ACKNOWLEDGMENTS}

Research supported by the 863 Program of the Ministry of Science and Technology of China (\#2006AA02Z198) and the National Basic Research and Development Program 973 of China (\#2009CB119000).

\section{REFERENCES}

Bae H, Bowers JH, Tooley PW and Bailey BA (2005). NEP1 orthologs encoding necrosis and ethylene inducing proteins exist as a multigene family in Phytophthora megakarya, causal agent of black pod disease on cacao. Mycol. Res. 109: 1373-1385.

Bailey BA (1995). Purification of a protein from culture filtrates of Fusarium oxysporm that induces ethylene and necrosis in leaves of Erythroloxylum coca. Phytopathology 85: 1250-1255.

Bailey BA, Bae H, Strem MD, Antunez de Mayolo G, et al. (2005). Developmental expression of stress response genes in Theobroma cacao leaves and their response to Nep1 treatment and a compatible infection by Phytophthora megakarya. Plant Physiol. Biochem. 43: 611-622.

Baldauf SL, Roger AJ, Wenk-Siefert I and Doolittle WF (2000). A kingdom-level phylogeny of eukaryotes based on combined protein data. Science 290: 972-977.

Dean RA, Talbot NJ, Ebbole DJ, Farman ML, et al. (2005). The genome sequence of the rice blast fungus Magnaporthe grisea. Nature 434: 980-986.

Fellbrich G, Romanski A, Varet A, Blume B, et al. (2002). NPP1, a Phytophthora-associated trigger of plant defense in parsley and Arabidopsis. Plant J. 32: 375-390.

Feng B, Li P, Wang H and Zhang X (2010). Functional analysis of Pcpme6 from oomycete plant pathogen Phytophthora capsici. Microb. Pathog. 49: 23-31.

Galagan JE, Calvo SE, Borkovich KA, Selker EU, et al. (2003). The genome sequence of the filamentous fungus Neurospora crassa. Nature 422: 859-868.

Galagan JE, Calvo SE, Cuomo C, Ma LJ, et al. (2005). Sequencing of Aspergillus nidulans and comparative analysis with A. fumigatus and A. oryzae. Nature 438: 1105-1115.

Garcia O, Macedo JA, Tiburcio R, Zaparoli G, et al. (2007). Characterization of necrosis and ethylene-inducing proteins (NEP) in the basidiomycete Moniliophthora perniciosa, the causal agent of witches' broom in Theobroma cacao. Mycol. Res. 111: 443-455.

Garzón-Ospina D, Cadavid LF and Patarroyo MA (2010). Differential expansion of the merozoite surface protein (msp)-7 gene family in Plasmodium species under a birth-and-death model of evolution. Mol. Phylogenet. Evol. 55: 399-408.

Gijzen M and Nurnberger T (2006). Nep1-like proteins from plant pathogens: recruitment and diversification of the NPP1 
domain across taxa. Phytochemistry 67: 1800-1807.

Jennings JC, Apel-Birkhold PC, Bailey BA and Anderson JD (2000). Induction of ethylene biosynthesis and necrosis in weed leaves by a Fusarium oxysporum protein. Weed Sci. 48: 7-14.

Lamour KH and Hausbeck MK (2004). Phytophthora capsici on vegetable crops: research progress and management challenges. Plant Dis. 88: 1292-1303.

Nei M and Rooney AP (2005). Concerted and birth-and-death evolution of multigene families. Annu. Rev. Genet. 39: 121-152.

Ottmann C, Luberacki B, Küfner I, Koch W, et al. (2009). A common toxin fold mediates microbial attack and plant defense. Proc. Natl. Acad. Sci. U. S. A. 106: 10359-10364.

Pare PW, Farag MA, Krishnamachari V, Zhang H, et al. (2005). Elicitors and priming agents initiate plant defense responses. Photosynth. Res. 85: 149-159.

Pemberton CL and Salmond GP (2004). The Nep1-like proteins-a growing family of microbial elicitors of plant necrosis. Mol. Plant. Pathol. 5: 353-359.

Qutob D, Kamoun S and Gijzen M (2002). Expression of a Phytophthora sojae necrosis-inducing protein occurs during transition from biotrophy to necrotrophy. Plant J. 32: 361-373.

Qutob D, Kemmerling B, Brunner F, Küfner I, et al. (2006). Phytotoxicity and innate immune responses induced by Nep1like proteins. Plant Cell 18: 3721-3744.

Tyler BM, Forster $\mathrm{H}$ and Coffey MD (1995). Inheritance of avirulence factors and restriction fragment length polymorphism markers in outcrosses of the oomycete Phytophthora sojae. Mol. Plant Microbe Interact. 8: 515-523.

Tyler BM, Tripathy S, Zhang X, Dehal P, et al. (2006). Phytophthora genome sequences uncover evolutionary origins and mechanisms of pathogenesis. Science 313: 1261-1266.

Wang JY, Cai Y, Gou JY, Mao YB, et al. (2004). VdNEP, an elicitor from Verticillium dahliae, induces cotton plant wilting. Appl. Environ. Microbiol. 70: 4989-4995.

\section{Supplementary Table 1}

Table S1. Candidate genes identified in the JGI Phytophthora capsici genome.

\begin{tabular}{lcc}
\hline Single copy genes & \multicolumn{2}{c}{ Multicopy genes } \\
\cline { 2 - 3 } & Genes & Identical members in genome \\
\hline 20406 & 23459 & 70850 \\
20844 & 70852 & 122619 \\
21024 & 76138 & 113086 \\
24573 & 82430 & 116399 \\
68295 & 114323 & 114326 \\
68503 & 7756 & $23286 ; 70849$ \\
69004 & 23292 & $7613 ; 37194$ \\
70605 & 71103 & $23660 ; 7723$ \\
72101 & 74207 & $27731 ; 27732 ; 74207$ \\
73591 & 8760 & $86961 ; 39481 ; 118625 ; 124767$ \\
75230 & 43883 & $43884 ; 43885 ; 43886 ; 43887 ; 66543 ;$ \\
78535 & & $91548 ; 91549 ; 91460 ; 91461 ; 91462 ;$ \\
78817 & & $119696 ; 41934 ; 41935 ; 41936 ; 41937 ;$ \\
82067 & & 65858 \\
86540 & &
\end{tabular}

